\title{
Spatial ordering of charge and spin in quasi-one-dimensional Wigner molecules
}

\author{
B. Szafran, ${ }^{1,2}$ F. M. Peeters, ${ }^{1}$ S. Bednarek, ${ }^{2}$ T. Chwiej, ${ }^{1,2}$ and J. Adamowski ${ }^{2}$ \\ ${ }^{1}$ Departement Natuurkunde, Universiteit Antwerpen (Campus Drie Eiken), B-2610 Antwerpen, Belgium \\ ${ }^{2}$ Faculty of Physics and Nuclear Techniques, AGH University of Science and Technology, al. Mickiewicza 30, 30-059 Kraków, Poland
}

(Received 19 January 2004; published 2 July 2004)

\begin{abstract}
Few-electron systems confined in quasi-one-dimensional quantum dots are studied by the configuration interaction approach. We consider the parity symmetry of states forming Wigner molecules in large quantum dots and find that for the spin-polarized Wigner molecules it strictly depends on the number of electrons. We investigate the spatial spin ordering in the inner coordinates of the quantum system and conclude that for small dots it has a short-range character and results mainly from the Pauli exclusion principle while the Wigner crystallization in large dots is accompanied by spin ordering over the entire length of the dot.
\end{abstract}

DOI: 10.1103/PhysRevB.70.035401

PACS number(s): 73.21.La, 73.20.Qt

\section{INTRODUCTION}

Strong confinement of charge carriers in two directions results in reduction of their degrees of freedom to a single one, i.e., in quasi-one-dimensional motion. Such onedimensional systems are realized typically in split-gate ${ }^{1,2}$ and cleaved-edge overgrowth ${ }^{3}$ semiconductor quantum wires, as well as in carbon nanotubes, ${ }^{4}$ but can also be realized in finite-size systems, i.e., in anisotropic quantum $\operatorname{dots}^{5}$ or quantum rings. ${ }^{6}$ There is a renewed interest in the onedimensional systems related to the recent progress of vapourliquid-solid fabrication of quantum wires of very high quality. ${ }^{7-9}$

The present paper is devoted to electron systems confined in one-dimensional quantum dots and in particular to their Wigner crystallization ${ }^{10}$ appearing when the electronelectron interaction dominates over the kinetic energy. Wigner electron solids (Wigner molecules) are predicted to appear in large dots ${ }^{11}$ or in strong magnetic fields. ${ }^{12}$ In the Wigner molecules the charge density separates into distinct charge maxima each corresponding to one of the confined electrons. Formation of Wigner molecules in the ground-state charge density in one-dimensional quantum dots was previously obtained in exact diagonalization ${ }^{13-15}$ and the density functional approach. ${ }^{16}$ In one-dimensional dots the Wigner localization appears in the laboratory frame, in contrast to the inner-coordinate crystallization appearing in circular quantum dots, ${ }^{12}$ including quantum rings. Transport properties of Wigner crystals formed in open infinite onedimensional systems have also been studied. ${ }^{17,18}$ The Luttinger liquid formalism has been applied ${ }^{19}$ to quantum wires with box-like boundary conditions, i.e., to the onedimensional quantum dots. Melting of classical onedimensional Wigner crystals has recently been described. ${ }^{20}$

We study the quasi-one-dimensional quantum dots using a configuration interaction approach with the effective electron-electron interaction potential which we derived recently. ${ }^{21}$ This work is a generalization of our exact twoelectron study ${ }^{15}$ to a larger number of electrons. In the weak confinement limit the ground state becomes nearly degenerate with respect to the spin configuration of the electron system. ${ }^{14,15}$ Similar approximate degeneracy has been found in quantum rings of large radius. ${ }^{22}$ In this paper we study the parity symmetry of the nearly degenerate states forming Wigner molecules in large dots. We show that for spinpolarized electrons the Wigner localization is formed only for one (even or odd) spatial parity of the state strictly dependent on the number of electrons. We present this dependence in the form of a theorem for which we provide a rigorous analytical proof. The found dependence of the parity of one-dimensional Wigner molecule states on the number of electrons is similar to the appearance of the magic angular momenta states for which Wigner crystallization is possible in circular dots. ${ }^{23,24}$ Furthermore, we discuss an inhibition of Wigner crystallization by a perturbation of the confinement potential through a central inversion-invariant potential well.

Magnetic spin ordering of electrons in one-dimensional space has been extensively studied ${ }^{25}$ in Hubbard models which, in one dimension with only nearest-neighbor hopping interactions, predict the appearance of a low-spin ground state. $^{26}$ This is a consequence ${ }^{26}$ of the Lieb-Mattis theorem ${ }^{27}$ which implies that without spin-dependent interactions the ground state of one-dimensional electron systems corresponds to the lowest possible spin quantum number $(S=0$ or $1 / 2$ ). This feature generally does not have to result in any spatial spin ordering. In this paper we use the exact numerical solution of the Schrödinger equation to investigate the spatial distribution of spins in the one-dimensional quantum dot and the relation between the charge and spatial spin ordering in the Wigner crystallization limit. We find that Wigner crystallization is accompanied by a long-range spinordering in the inner coordinates of the system instead of a spin-symmetry breaking predicted by the density functional theory. ${ }^{16,28}$ In the ground-state this ordering has a clear antiferromagnetic character.

This paper is organized as follows. In Sec. II we present the theoretical method. Section III contains the results for the Wigner localization and ground state degeneracy of the few electron systems. In Sec. IV we present proof for the dependence of the parity of spin-polarized Wigner molecules on the number of electrons. Section V contains a discussion of the effect of a central defect on Wigner crystallization. In Sec. VI the study of spin ordering is presented. Section VII contains our summary and conclusions. 

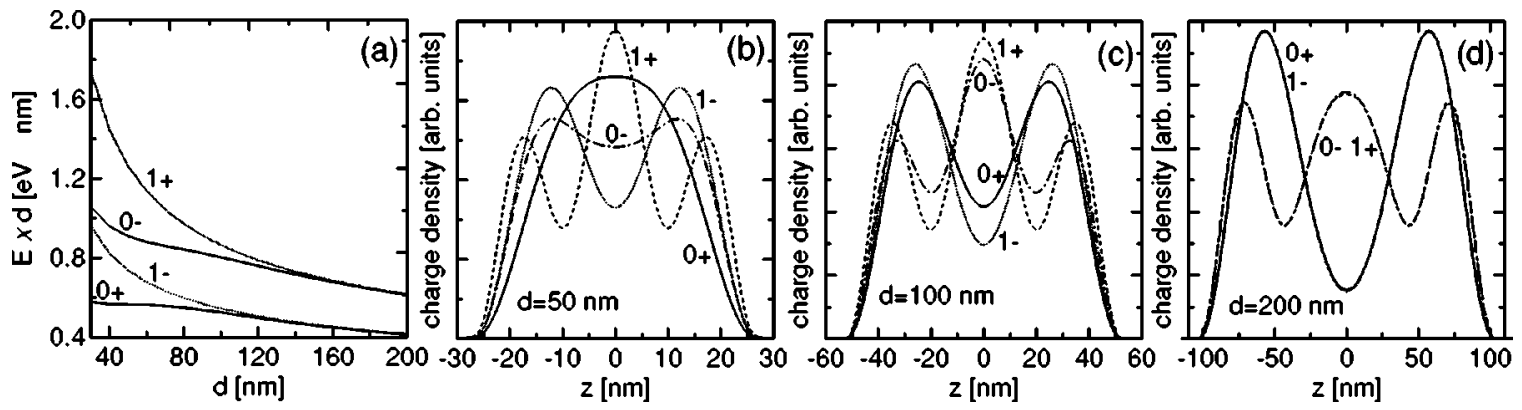

FIG. 1. (a) Lowest energy levels multiplied by the dot length for $N=2$. Numbers close to the curves denote the total spin quantum number of the corresponding states and signs,+- stand for even and odd parity symmetry. (b), (c), (d) Charge density of $0+, 1-, 1+$, and $0-$ states plotted with solid, dotted, dashed, and dash-dotted lines for $d=50,100$, and $200 \mathrm{~nm}$, respectively.

\section{THEORY}

We consider $N$ electrons confined in a quasi-onedimensional quantum dot with strong lateral harmonicoscillator confinement potential. The Hamiltonian of the system reads

$$
H=\sum_{i=1}^{N} h_{i}+\sum_{i=1}^{N} \sum_{j>i}^{N} \frac{\kappa}{r_{i j}},
$$

where $h$ stands for the single-electron Hamiltonian

$$
h=-\frac{\hbar^{2}}{2 m^{*}} \nabla^{2}+\frac{m^{*} \omega^{2}}{2}\left(x^{2}+y^{2}\right)+V(z),
$$

$V(z)$ is the confinement potential in the $z$ direction. For a large lateral harmonic-oscillator confinement energy $(\hbar \omega)$ the movement of electrons in the $(x, y)$ plane is frozen to the harmonic-oscillator ground state. Then, one can perform integration $^{21}$ over the lateral degrees of freedom which results in the following Hamiltonian:

$$
\begin{aligned}
H= & N \hbar \omega+\sum_{i=1}^{N} h_{i}^{1 D}+\sum_{i=1}^{N} \sum_{j>i}^{N}(\pi / 2)^{1 / 2}(\kappa / l) \\
& \times \operatorname{erfc}\left(z_{i j} / 2^{1 / 2} l\right) \exp \left(z_{i j}^{2} / 2 l^{2}\right),
\end{aligned}
$$

where $z_{i j}=\left|z_{i}-z_{j}\right|$ and

$$
h^{1 D}=-\frac{\hbar^{2}}{2 m^{*}} \frac{d^{2}}{d z^{2}}+V(z)
$$

is the single-electron one-dimensional Hamiltonian. In the following we will neglect the first term in Eq. (3), i.e., the lateral confinement energy which is independent of the form of wave functions in the $z$ direction. The last term in Eq. (3) is the effective interaction energy ${ }^{21}$ for electrons in a quasione-dimensional environment resulting from integration of the Coulomb potential over the lateral coordinates, $m^{*}$ is the effective mass, $\kappa=e^{2} / 4 \pi \varepsilon_{0} \varepsilon$, $\varepsilon$ is the dielectric constant, and $l=\sqrt{\hbar / m^{*}} \omega$. We assume $V(z)=V_{\text {well }}(z)$, a rectangular potential well of depth $V_{0}=200 \mathrm{meV}$, and width $d$. We adopt GaAs material parameters, i.e., $m^{*}=0.067 m_{e 0}, \epsilon=12.4$ as well as $\hbar \omega=10 \mathrm{meV}(l=10.66 \mathrm{~nm})$ for the lateral confinement energy. Calculations have been performed for $N=2, \ldots, 5$ electrons by the configuration interaction approach with a basis set of Slater determinants built with single-electron spin or- bitals. Spatial single-electron wave functions have been obtained by numerical diagonalization of the finite-difference version of the single-electron one-dimensional Hamiltonian (4) on a mesh of points. In construction of the Slater determinants with required spin and parity symmetries we use the spatial wave functions of up to eight lowest-energy singleelectron states which results in a Slater determinant basis size of up to 1520 elements and an accuracy better than $0.01 \mathrm{meV}$.

The present approach is based on the assumption that only the lowest state of the lateral $(x, y)$ quantization is occupied. We performed test calculations for two, three and four electrons to check the validity of this approach. We allowed the electrons to occupy also the $p$-type lowest excited state of the lateral quantization with angular momentum $\pm \hbar$. Inclusion of $p$ states not only allows for determination of the critical well length above which the $p$ shell is emptied, but it is also helpful to estimate the importance of the angular correlations in the $x-y$ plane. The Coulomb matrix elements were evaluated using effective interaction potentials derived with the use of the Fourier transform technique. ${ }^{21}$ We have obtained the following results: the $p$ shell is left empty for $d>39$ and $41 \mathrm{~nm}$ for $N=3$ and 4 , respectively (for two electrons the $p$ shell is never occupied). Accounting for the $x-y$ correlations via inclusion of the $p$-type orbitals in the configuration interaction basis lowers the two-electron total energy estimates by $0.18,0.12,0.01$, and $10^{-4} \mathrm{meV}$ for $d=40,50,100$, and $200 \mathrm{~nm}$, respectively. These "lateral correlation energies" for the same values of $d$ are equal to $0.4,0.3,0.08$, and 4 $\times 10^{-3} \mathrm{meV}$ for $N=3$, and $1.18,0.67,0.23$, and 0.03 for $N$ $=4$, respectively. The energy overestimation in the range of $d$ studied further is never significant and the present approach is nearly exact in the Wigner localization regime.

\section{GROUND STATE DEGENERACY AND WIGNER CRYSTALLIZATION}

In this paper we label the states by their total spin $S$ and parity quantum numbers using the notation: $S \pm$, where the positive (negative) sign stands for even (odd) parity. We discuss only the lowest-energy states for a given spin-orbital symmetry. Figure 1(a) shows the lowest energy levels of the two-electron system multiplied by the dot length $d$ as functions of $d$. For large dots the states $0+$ and $1-$ as well as 

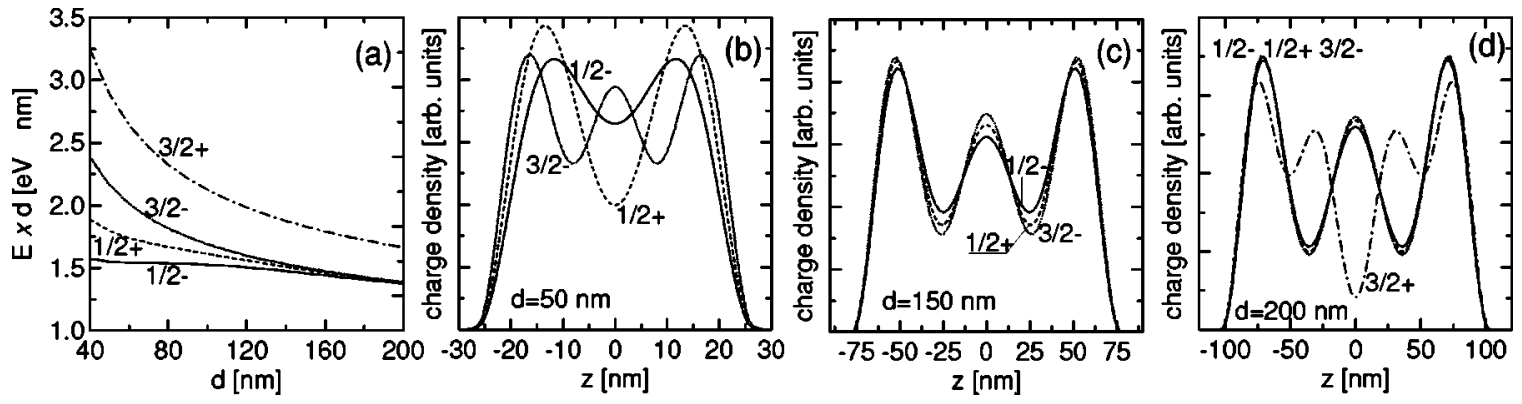

FIG. 2. (a) Lowest energy levels multiplied by the dot length for $N=3$. (b), (c), (d) Charge density of $1 / 2-, 1 / 2+$, and $3 / 2-$ states plotted with solid, dashed, and dotted lines for $d=50,150$, and $200 \mathrm{~nm}$, respectively. In (d) the charge density of the $3 / 2+$ state is shown by the dash-dotted curve.

$0-$ and $1+$ become mutually degenerate. For large values of $d$ potential energy related to penetration of electrons into the barrier region is negligible, the kinetic energy scales as $1 / d^{2}$ and the Coulomb energy as $1 / d$. Therefore, the product of energy and dot length for large $d$ behaves as $f(d)=C+D / d$ function, where the constants $C$ and $D$ are related to the Coulomb and kinetic energy, respectively. The energy levels of the degenerate pairs of states tend to different constants in the infinite $d$ limit which is apparently due to different values of the Coulomb interaction in these pairs of states. The evolution of the charge density for growing length of the dot is shown in Figs. 1(b)-1(d). For large dots [cf. Fig. 1(d)] the charge densities of the degenerate pairs of states become identical. In the ground state the charge density has two pronounced maxima which indicates the separation of electron charges into two charge islands, i.e., the Wigner crystallization. Figure 1 shows that the singlet-triplet degeneracy obtained previously ${ }^{15}$ for the two-electron ground-state appears also in the first excited state.

Figure 2(a) shows the energy levels and Figs. 2(b)-2(d) the charge density for the lowest-energy states of the threeelectron system for increasing $d$. For three electrons the Wigner molecule is formed in states $1 / 2-, 1 / 2+$, and $3 / 2-$ which become degenerate for large $d$. In the state $3 / 2+$ the charge density exhibits four maxima [cf. Fig. 2(d)], which apparently prevents this state to be degenerate with the ground state.

In the four-electron system the ground state corresponds to $0+$ symmetry. The states $1-, 1+$, and $2+$ for large dots [cf. Fig. 3(a)] tend to the degeneracy with the ground state. The charge densities of these states for large dots present four distinct maxima [cf. Fig. 3(d)]. Energy levels corresponding to states $0-$ and 2- are separated by a significant energy distance from the ground state [cf. Fig. 3(a)] and in large dots they correspond to identical charge densities with five maxima. The ground state charge density evolution obtained for $N=3$ and 4 is in a qualitative agreement with the results of Ref. 13.

Finally, in the five-electron system the ground state of $1 / 2+$ symmetry becomes degenerate with $1 / 2-, 3 / 2+$, $3 / 2-$, and $5 / 2+$ states [cf. Fig. 4(a)] forming Wigner molecules for large dots [cf. Figs. 4(b)-4(d)]. The spin polarized state of odd parity 5/2- does not become degenerate with the ground-state and its charge density in large dots forms six maxima [cf. Fig. 4(d)].

In the entire $d$ range and for all electron numbers studied the order of the lowest energy levels for given total spin quantum numbers (neglecting the parity) follow the order of the spin quantum numbers, which is in agreement with the theorem of Lieb and Mattis. ${ }^{27}$ In large dots the ground-state degeneracy appears. In Ref. 14 the degeneracy was interpreted in terms of a vanishing tunnel coupling between the local minima of the total $\mathrm{N}$-dimensional potential energy. The present results indicate that the nearly degenerate states possess the same charge density in the laboratory frame. Moreover, we observe the following regularities. In the limit of Wigner localization the ground state of the $N$-electron system appears for $N$ different pairs of the spin and parity quantum numbers. ${ }^{29}$ For even electron numbers $N=2$ and 4 , $N$ charge maxima are formed only for even parity states with
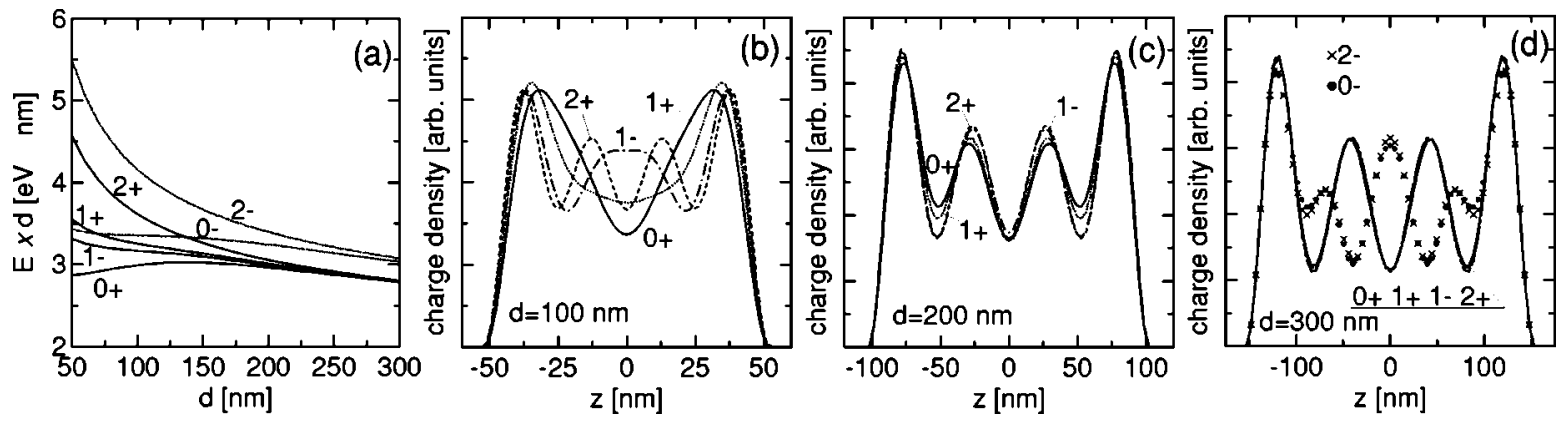

FIG. 3. (a) Four-electron energy levels multiplied by the dot length. (b), (c), (d) Charge density of 0+, 1-, 1+, and 2+ four-electron states plotted with solid, dash-dotted, dotted, and dashed lines for $d=100,200$, and $300 \mathrm{~nm}$, respectively. In (d) the charge densities of 2- and $0+$ states are marked with crosses and dots, respectively. 

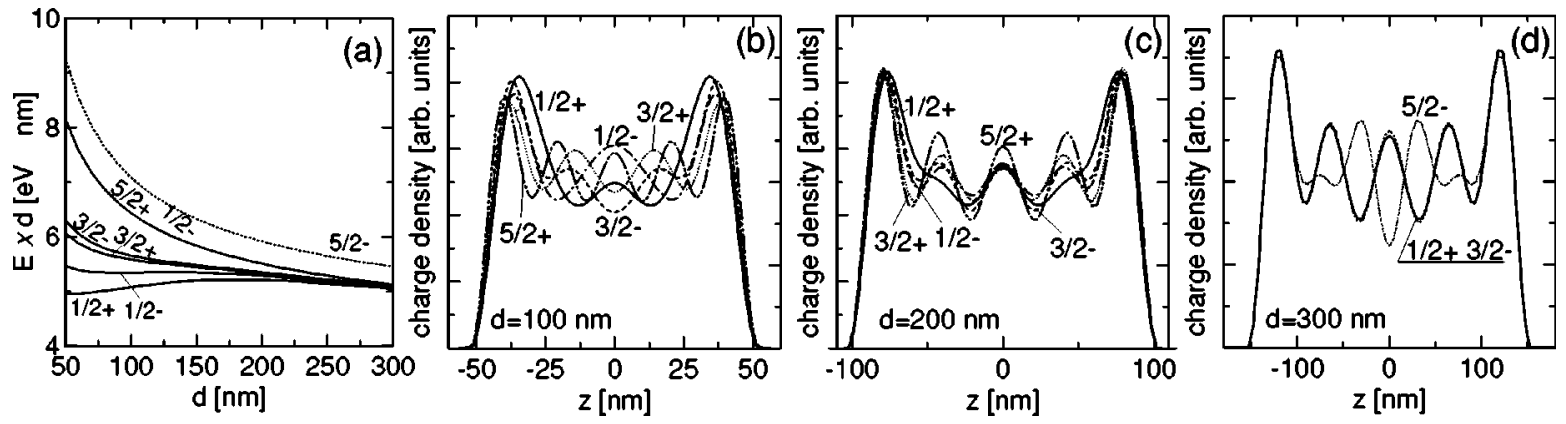

FIG. 4. (a) Five-electron energy levels multiplied by the dot length. Even (odd) parity levels are plotted with solid (dotted) lines. (b), (c) Charge density of $1 / 2+, 1 / 2-, 3 / 2+, 3 / 2-$, and $5 / 2+$ states plotted with solid, dash-dotted, dotted, dashed, and dash-double-dot lines for $d=100$ and $200 \mathrm{~nm}$, respectively. In (d) the charge density of the $1 / 2+, 3 / 2-$, and $5 / 2-$ state is shown by solid, dashed, and dotted lines, respectively [charge densities of $1 / 2-, 3 / 2+$, and 5/2+ are almost identical with the $1 / 2+$ and $3 / 2-$ charge densities are therefore omitted in (d) for the sake of clarity].

$S=0$, while the odd parity zero-spin states possess $N+1$ charge maxima [cf. Figs. 1(d) and 3(d)]. The spin-polarized Wigner-localized state can only be formed for one (even or odd) parity. Namely, the parity of the spin-polarized Wigner molecule state is even for four and five electrons and odd for two and three electrons. The charge density of the spinpolarized state of the other parity exhibits $N+1$ maxima, i.e., the state does not form a Wigner molecule and as a consequence does not become degenerate with the ground state even for large dots. This conclusion will be cast into a theorem in the next section.

\section{PARITY OF SPIN-POLARIZED WIGNER MOLECULE STATES}

Here we give an analytical proof of the theorem: for an odd number of electrons $N=2 M+1$ as well as for an even number of electrons $N=2 M$ the parity of one-dimensional spin-polarized Wigner-molecule state is even (odd) for even (odd) value of the integer $M$.

We will present here the proof for an odd number of electrons (the proof for even $N$ can be easily deduced from the present demonstration). For odd $N$ one of the electrons resides near the center of the dot (point $z_{0}=0$, cf. Fig. 5), and the others occupy spatially symmetric sites to the left and

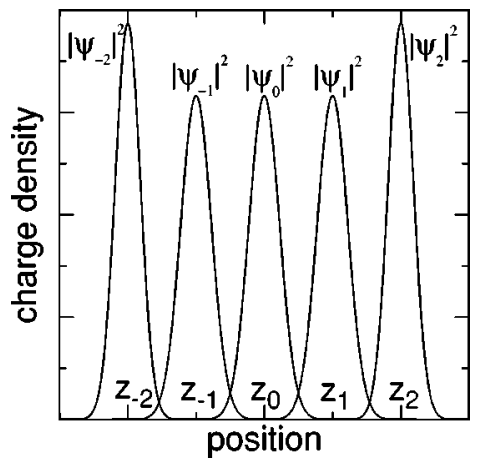

FIG. 5. Illustration to the proof that for $N=2 M+1$ or $N=2 M$ electrons the parity of the spin-polarized state which exhibits Wigner localization is accordant with the parity of $M$. right of the dot around points which satisfy $z_{k}=-z_{-k}$ for $k$ $= \pm 1, \pm 2, \ldots, \pm M$. In the Wigner phase the total charge density possesses $N$ maxima corresponding to the separate single-electron charge densities. A single-electron density $\left|\psi_{k}(z)\right|^{2}$ is localized around point $z_{k}$. In the Wigner limit the overlap between the single-electron charge densities vanishes (the proof is only valid when this overlap is negligible), so the total charge density can be expressed as their sum. Since the total charge density is symmetric with respect to the origin the following equality holds:

$$
\left|\psi_{k}(-z)\right|^{2}=\left|\psi_{-k}(z)\right|^{2}
$$

which results in the following relation for the single-electron wave functions:

$$
\psi_{k}(-z)=e^{i \phi_{k}} \psi_{-k}(z)
$$

where the phase $\phi_{k}$ is a real number. Relation (6) with changed sign of $k$ reads:

$$
\psi_{-k}(-z)=e^{i \phi_{-k}} \psi_{k}(z)
$$

Phases $\phi_{k}$ and $\phi_{-k}$ are not independent. Changing the sign of $z$ in Eq. (7) and making use of relation (6) we arrive at

$$
\psi_{-k}(z)=e^{i \phi_{-k}} \psi_{k}(-z)=e^{i\left(\phi_{-k}+\phi_{k}\right)} \psi_{-k}(z),
$$

hence,

$$
\phi_{k}=-\phi_{-k}
$$

up to an unimportant multiple of $2 \pi$. Considering relation (6) for $k=0$ and reminding that we arrive at the same value $\psi_{0}(0)$ (nonzero for odd $N$ ) approaching the origin from both positive and negative sides we arrive at $\phi_{0}=0$ and consequently $\psi_{0}$ is an even parity function 


$$
\psi_{0}(-z)=\psi_{0}(z)
$$

Since the considered state is spin polarized the spin and spatial parts of the wave function can be separated into a product

$$
\begin{aligned}
\chi\left(z_{1}, \sigma_{1}, \ldots, z_{N}, \sigma_{N}\right)= & \alpha\left(\sigma_{1}\right) \alpha\left(\sigma_{2}\right) \cdots \alpha\left(\sigma_{N}\right) \\
& \times \Psi\left(z_{1}, z_{2}, \ldots, z_{N}\right),
\end{aligned}
$$

where $\alpha$ is an eigenfunction of the single-electron spin $z$-component operator. The spatial wave function $\Psi$ can be written as a Slater determinant ${ }^{30}$

$$
\begin{aligned}
& \Psi\left(z_{1}, z_{2}, \ldots, z_{N}\right) \\
& =\left|\begin{array}{cccc}
\psi_{-M}\left(z_{1}\right) & \psi_{-M+1}\left(z_{1}\right) & \ldots \psi_{M-1}\left(z_{1}\right) & \psi_{M}\left(z_{1}\right) \\
\psi_{-M}\left(z_{2}\right) & \psi_{-M+1}\left(z_{2}\right) & \ldots \psi_{M-1}\left(z_{2}\right) & \psi_{M}\left(z_{2}\right) \\
\ldots & & & \\
\psi_{-M}\left(z_{N}\right) & \psi_{-M+1}\left(z_{N}\right) & \ldots \psi_{M-1}\left(z_{N}\right) & \psi_{M}\left(z_{N}\right)
\end{array}\right| .
\end{aligned}
$$

We apply the parity operator on $\Psi$ and make use of properties (6) and (9) obtaining

$$
\Psi\left(-z_{1},-z_{2}, \ldots,-z_{N}\right)=\left|\begin{array}{cccc}
e^{-i \phi_{M}} \psi_{M}\left(z_{1}\right) & e^{-i \phi_{M-1}} \psi_{M-1}\left(z_{1}\right) & \ldots e^{i \phi_{M-1}} \psi_{-M+1}\left(z_{1}\right) & e^{i \phi_{M}} \psi_{-M}\left(z_{1}\right) \\
e^{-i \phi_{M}} \psi_{M}\left(z_{2}\right) & e^{-i \phi_{M-1}} \psi_{M-1}\left(z_{2}\right) & \ldots e^{i \phi_{M-1}} \psi_{-M+1}\left(z_{2}\right) & e^{i \phi_{M}} \psi_{-M}\left(z_{2}\right) \\
\ldots & & & \\
e^{-i \phi_{M}} \psi_{M}\left(z_{N}\right) & e^{-i \phi_{M-1}} \psi_{M-1}\left(z_{N}\right) & \ldots e^{i \phi_{M-1}} \psi_{-M+1}\left(z_{N}\right) & e^{i \phi_{M}} \psi_{-M}\left(z_{N}\right)
\end{array}\right|
$$

Phase factors can be extracted from each of the determinant columns, which yields

$$
\begin{aligned}
& \Psi\left(-z_{1},-z_{2}, \ldots,-z_{N}\right) \\
& =e^{-i\left(\phi_{M}+\phi_{M-1}+\ldots+\phi_{-M+1}+\phi_{-M}\right)} \\
& \times\left|\begin{array}{cccc}
\psi_{M}\left(z_{1}\right) & \psi_{M-1}\left(z_{1}\right) & \ldots \psi_{-M+1}\left(z_{1}\right) & \psi_{-M}\left(z_{1}\right) \\
\psi_{M}\left(z_{2}\right) & \psi_{M-1}\left(z_{2}\right) & \ldots \psi_{-M+1}\left(z_{2}\right) & \psi_{-M}\left(z_{2}\right) \\
\ldots & & & \\
\psi_{M}\left(z_{N}\right) & \psi_{M-1}\left(z_{N}\right) & \ldots \psi_{-M+1}\left(z_{N}\right) & \psi_{-M}\left(z_{N}\right)
\end{array}\right| .
\end{aligned}
$$

The phases in front of the determinant in Eq. (14) cancel according to property (9). Exchanging $M$ pairs of corresponding columns in the determinant we arrive at Eq. (12) but multiplied by $(-1)^{M}$, which proofs that the parity of spinpolarized one-dimensional Wigner molecule state is determined by the odd or even value of $M$.

We have found that two- and four-electron zero-spin states can form a Wigner-localized charge density only for even spatial parity. We are unable to proof in general that the zero-spin state with Wigner localization has to be of even parity for even $N$. But for $N=2$ such a proof is easily given. In this case the spin and spatial parts of the wave function can be separated as follows:

$$
\begin{aligned}
\chi^{0+}\left(z_{1}, \sigma_{1}, z_{2}, \sigma_{2}\right)= & {\left[\alpha\left(\sigma_{1}\right) \beta\left(\sigma_{2}\right)-\alpha\left(\sigma_{2}\right) \beta\left(\sigma_{1}\right)\right] } \\
& \times\left[\psi_{1}\left(z_{1}\right) \psi_{-1}\left(z_{2}\right)+\psi_{-1}\left(z_{1}\right) \psi_{1}\left(z_{2}\right)\right] .
\end{aligned}
$$

Applying the parity operator to the spatial part of this wave function and making use of the properties of the singleelectron wave functions given earlier we find that this wave function is of even parity. Moreover, it follows that construction of a symmetric spatial wave function for odd-parity sin- glet (zero-spin) two-electron states (0-) requires at least three single-electron functions, for instance, the function

$$
\begin{aligned}
\Psi^{0-}\left(z_{1}, z_{2}\right)= & \psi_{0}\left(z_{1}\right) \psi_{1}\left(z_{2}\right)+\psi_{1}\left(z_{1}\right) \psi_{0}\left(z_{2}\right)-\psi_{0}\left(z_{1}\right) \psi_{-1}\left(z_{2}\right) \\
& -\psi_{-1}\left(z_{1}\right) \psi_{0}\left(z_{2}\right),
\end{aligned}
$$

is of odd parity provided that we take zero phase shifts in relation (6). Indeed, the $0-$ state for $N=2$ exhibits three charge maxima [see Fig. 1(d)]. Moreover, construction of a triplet antisymmetric spatial wave function with even parity $(1+)$ also requires at least three localized functions, for instance

$$
\begin{aligned}
\Psi^{1+}\left(z_{1}, z_{2}\right)= & \psi_{0}\left(z_{1}\right) \psi_{1}\left(z_{2}\right)-\psi_{1}\left(z_{1}\right) \psi_{0}\left(z_{2}\right)+\psi_{0}\left(z_{1}\right) \psi_{-1}\left(z_{2}\right) \\
& -\psi_{-1}\left(z_{1}\right) \psi_{0}\left(z_{2}\right)
\end{aligned}
$$

possesses the required symmetries for zero phase shifts in relation (6). The charge density corresponding to wave functions (16) and (17) is the same provided that the overlaps between the functions $\psi_{i}$ are negligible. Figure 1(d) shows that the charge densities of the states $0-$ and $1+$ are indeed indistinguishable. The area below the central maximum of the probability density of degenerate $0-$ and $1+$ states in Fig. 1(d) is two times larger than the area below each of the extreme maxima, which can be interpreted by saying that one of the electrons stays in the neighborhood of the center of the system with $100 \%$ probability while probabilities of finding the other one at the left or right end of the well are equal to $50 \%$. This feature is in agreement with the probability amplitudes (16) and (17). Although in the wave functions (16) and (17) the electron positions are separated, this separation has a nonclassical character since the charge maxima at the left and right ends of the dot correspond to subelectron charges. Therefore, we do not refer to this separation as Wigner localization. Average electron-electron distances in states described by wave functions (16) and (17) are smaller 

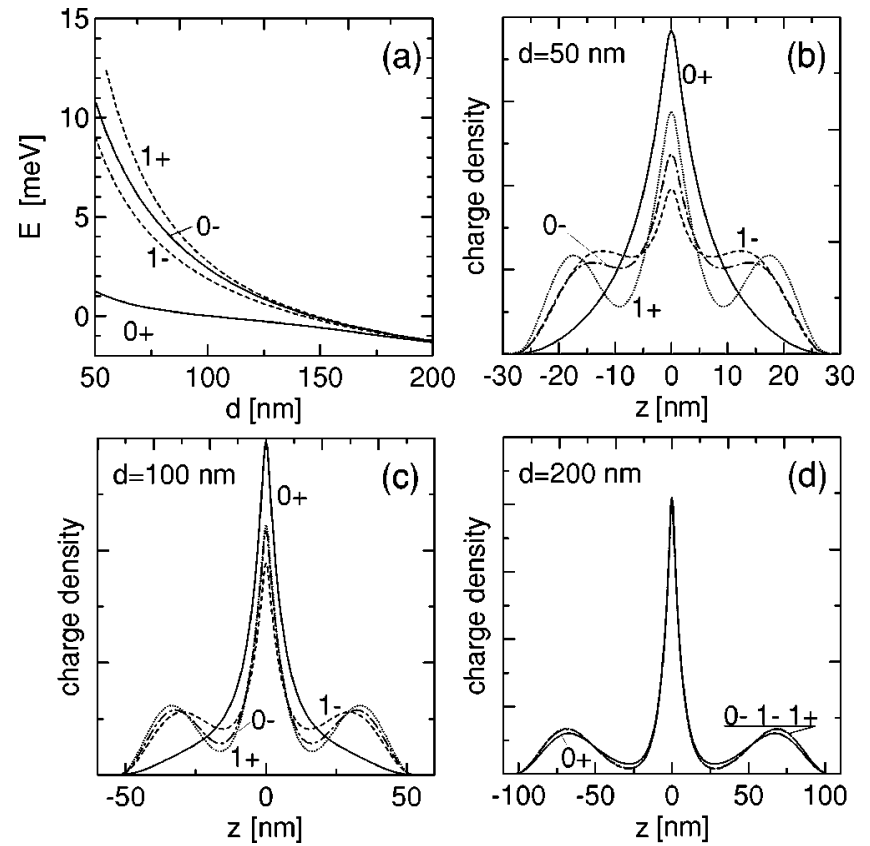

FIG. 6. (a) Lowest energy levels for $N=2$ as functions of the length of the well with a central attractive cavity [Eq. (18)]. Numbers close to the curves denote the total spin quantum number of the corresponding states and signs,+- stand for even and odd parity symmetry, respectively. (b), (c), (d) Charge density of $0+, 1-, 1+$, and 0 - states plotted with solid, dotted, dashed, and dash-dot curves for $d=50,100$, and $200 \mathrm{~nm}$, respectively.

than in states $0+, 1-$ with two charge maxima, which leads to a larger value of the Coulomb interaction energy and consequently to an energy separation between pairs of degenerate states $0+, 1-$ and $0-, 1+$ presented in Fig. 1(a) in the weak confinement limit.

\section{WIGNER CRYSTALLIZATION IN THE PRESENCE OF A DEFECT POTENTIAL}

The presence of defects can significantly perturb the Wigner crystallization in large systems. We consider here a thin attractive cavity just deep enough to bind one electron. The perturbed quantum dot potential is of the form

$$
V(z)=V_{\text {well }}(z)+V_{\text {defect }}(z),
$$

where $V_{\text {defect }}(z)=-50 \mathrm{meV}$ for $|z|<1 \mathrm{~nm}$ and $V_{\text {defect }}(z)=0$ for $|z|>1 \mathrm{~nm}$. The assumption that the defect is localized in the center of the system does not perturb the inversion invariance of the total potential.

Figure 6(a) shows that contrary to the unperturbed quantum well potential [cf. Fig. 1(a)] the $0-$ and $1+$ states become degenerate with the $0+$ and $1-$ states. Figures 6(b)-6(d) show the evolution of the charge densities of the four considered states with increasing size of the system. For large well thickness [cf. Fig. 1(d)] the charge densities of these states become indistinguishable. One of the electrons is trapped by the potential of the central cavity which results in the sharp central peak of the charge density. The probabilities to find the other electron at the left or right side of the origin
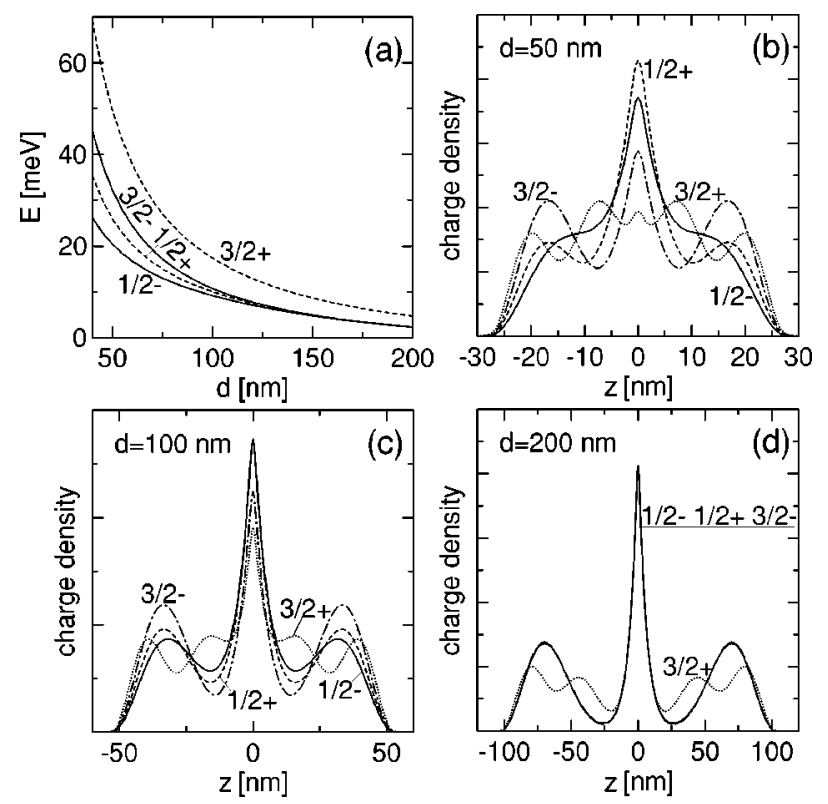

FIG. 7. (a) Lowest energy levels for $N=3$ as function of the length of the well with a central attractive cavity [Eq. (18)]. Numbers close to the curves denote the total spin quantum number of the corresponding states and signs,+- stand for even and odd parity symmetry. (b),(c),(d) Charge density of $0+, 1-, 1+$, and $0-$ states plotted with solid, dotted, dashed, and dash-dot curves for $d=50$, 100 , and $200 \mathrm{~nm}$, respectively.

are equal. This differs essentially from the two-electron Wigner molecule charge density in the unperturbed dot [cf. Fig. 1], for which the probability to find an electron in the center of the well was negligible and for which each of the two charge maxima could be associated with an integer electron charge. The formation of three maxima in the charge density is possible for all states [cf. Eqs. (16) and (17), for 0 - and 1+ states, similar formulas can be given for the other two]. Therefore, the ground state tends to a fourfold degeneracy in contrast to the double degeneracy for the unperturbed dot [cf. Fig. 1(a)].

Figure 7 shows the lowest energy levels and corresponding charge density evolution for the three-electron system. Contrary to the two-electron system the central defect does not perturb the number of charge maxima, Wigner localization appears similarly as for the unperturbed dot [cf. Fig. 2] for $1 / 2+, 1 / 2-$, and $3 / 2-$ states which become degenerate in the Wigner localization limit. State $3 / 2+$, which according to the theorem given in Sec. IV cannot form a Wigner phase lies higher in energy, like for the unperturbed dot.

The influence of the central attractive defect is qualitatively different for odd and even electron number. For an odd number of electrons it simply enhances the localization of the central electron, and does not influence the ground state degeneracy. While for even $N$ it destroys Wigner crystallization leading to the appearance of an extra charge maximum corresponding to subelectron charge and allows more states to become degenerate with the ground state.

\section{SPATIAL SPIN ORDERING IN THE WIGNER LIMIT}

It is interesting to look whether the low-spin ground states exhibit any spatial antiferromagnetic ordering of the electron 

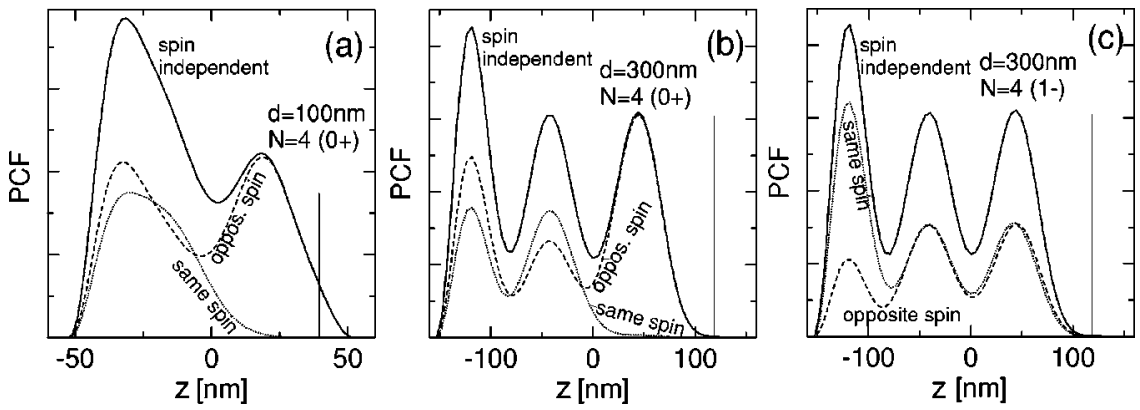

FIG. 8. PCFs for four electrons in state $0+[(\mathrm{a}),(\mathrm{b})]$ and state 1 - with $S_{z}=\hbar$ (c) for $d=100$ (a) and $300 \mathrm{~nm}[(\mathrm{~b}),(\mathrm{c})]$. One of the electrons is fixed and its position is marked by a thin vertical line. Solid curves show the spinindependent PCF, dashed (dotted) curves show the opposite (same) spin PCF. spins. For even number of electrons and arbitrary dot length the ground state corresponds to zero total spin. In this case the spin-up and spin-down densities are exactly equal to each other, so that spin ordering is not visible in the laboratory frame of reference. In order to investigate a possible spin ordering in the zero-spin ground states one has to look into the inner coordinates of the quantum system. We use here the spin-dependent pair correlation functions (PCFs) defined for a given state by the expectation values

$$
\begin{aligned}
F_{\mathrm{PCF}}^{\mathrm{same}}\left(z_{a}, z_{b}\right)= & \left\langle\sum_{i=1}^{N} \sum_{j>i}^{N} \delta\left(z_{a}-z_{i}\right) \delta\left(z_{b}-z_{j}\right)\right. \\
& \times\left(\left|\alpha\left(\sigma_{i}\right) \alpha\left(\sigma_{j}\right)\right\rangle\left\langle\alpha\left(\sigma_{i}\right) \alpha\left(\sigma_{j}\right)\right|\right. \\
& \left.\left.+\left|\beta\left(\sigma_{i}\right) \beta\left(\sigma_{j}\right)\right\rangle\left\langle\beta\left(\sigma_{i}\right) \beta\left(\sigma_{j}\right)\right|\right)\right\rangle,
\end{aligned}
$$

and

$$
\begin{aligned}
F_{\mathrm{PCF}}^{\mathrm{oppo}}\left(z_{a}, z_{b}\right)= & \left\langle\sum_{i=1}^{N} \sum_{j>i}^{N} \delta\left(z_{a}-z_{i}\right) \delta\left(z_{b}-z_{j}\right)\right. \\
& \times\left(\left|\alpha\left(\sigma_{i}\right) \beta\left(\sigma_{j}\right)\right\rangle\left\langle\alpha\left(\sigma_{i}\right) \beta\left(\sigma_{j}\right)\right|\right. \\
& \left.\left.+\left|\beta\left(\sigma_{i}\right) \alpha\left(\sigma_{j}\right)\right\rangle\left\langle\beta\left(\sigma_{i}\right) \alpha\left(\sigma_{j}\right)\right|\right)\right\rangle,
\end{aligned}
$$

where $\alpha$ and $\beta$ stand for spin-up and spin-down eigenstates, respectively. Functions (19) and (20) give the probability of finding at positions $z_{a}$ and $z_{b}$ a pair of electrons with the same (19) or opposite (20) spins. The sum of functions (19) and (20) gives the spin-independent PCF.

Figure 8(a) shows the PCF plots for the four-electron ground state in a small quantum dot [cf. Fig. 3(b)] with $d$ $=100 \mathrm{~nm}$. The position of one of the electrons is fixed near the right end of the dot [position marked by the thin vertical line in Fig. 8(a)]. We see that the probability of finding an electron with the same spin in the neighborhood of the fixedposition electron is zero, which is a signature of the Pauli exclusion principle. At the left side of the dot probabilities of finding an electron with the same or opposite spin as the one of the fixed position electron are nearly equal. For the total zero-spin states in relatively small dots the spin ordering in the inner coordinates is of short range and results from the Pauli exclusion. We only found a long-range innercoordinate spin ordering in the Wigner crystallization limit. Figure 8(b) shows the plot for the four-electron ground state with $d=300 \mathrm{~nm}$. The charge density of the system exhibits four distinct maxima [cf. Fig. 3(d)]. We fix the position of one of the electrons at the rightmost density maximum [cf. the vertical line in Fig. 8(b)]. The probability that the electron in the adjacent maximum has the opposite spin is nearly $100 \%$. The spin-dependent PCFs also differ for the two charge maxima at the left of the origin. An electron confined at the first (second) charge maximum to the left of the origin is more probable to have the same (opposite) spin as the one of the fixed electron. The ordering is of a probabilistic character, so that the antiferromagnetic order of spins is the most probable to be found, but the probability is not $100 \%$. The spin ordering in this state has a clearly antiferromagnetic character and its range covers the entire length of the dot. A similar inner-coordinate antiferromagnetic order was previously found for quantum rings. ${ }^{6}$

The $100 \%$ probability of finding the opposite spin in the charge maximum adjacent to the maximum associated with the fixed electron presented in Fig. 8(b) is not, as one could naively expect, related to the Pauli exclusion. In Fig. 8(c) we plotted the PCF for the 1- state, which becomes degenerate with the ground $0+$ state in the weak confinement limit. We see that in this state the spin of electrons confined in the two central maxima is independent of the spin of the electron at the rightmost maximum. However, in this state one may expect that the electrons at the opposite ends of the dot have the same spin, which means that also in this state a longrange spin ordering exists, even if it is not of antiferromagnetic origin.

For odd number of electrons the difference between spin-up and spin-down densities appears in the laboratory frame. This is qualitatively different from quantum rings, which in fact are endless structures. Figure 9(a) shows the spin densities for a relatively small dot length of $d$ $=100 \mathrm{~nm}$ [too small for the ground state Wigner localization to appear, cf. Fig. 4(b)]. The spin-up electrons tend to gather at the extreme left and right ends of the dot as well as in its center. The spin-down density is minimal in the center of the dot, and the overall spin density (difference of the spin-up and spin-down densities) exhibits antiferromagnetic sign oscillations within the dot. These sign oscillations are due to the electron-electron interaction since in the noninteracting electron system the majority spin-up density is nowhere smaller than the spin-down density. For larger systems $[d$ $=250 \mathrm{~nm}$, cf. Fig. 9(b)] the antiferromagnetic spin oscillations become more pronounced. However, for even larger $d$ [cf. Figs. 9(c) and 9(d)], for which the Wigner molecule appear in the $1 / 2+$ ground state, the typically antiferromagnetic 

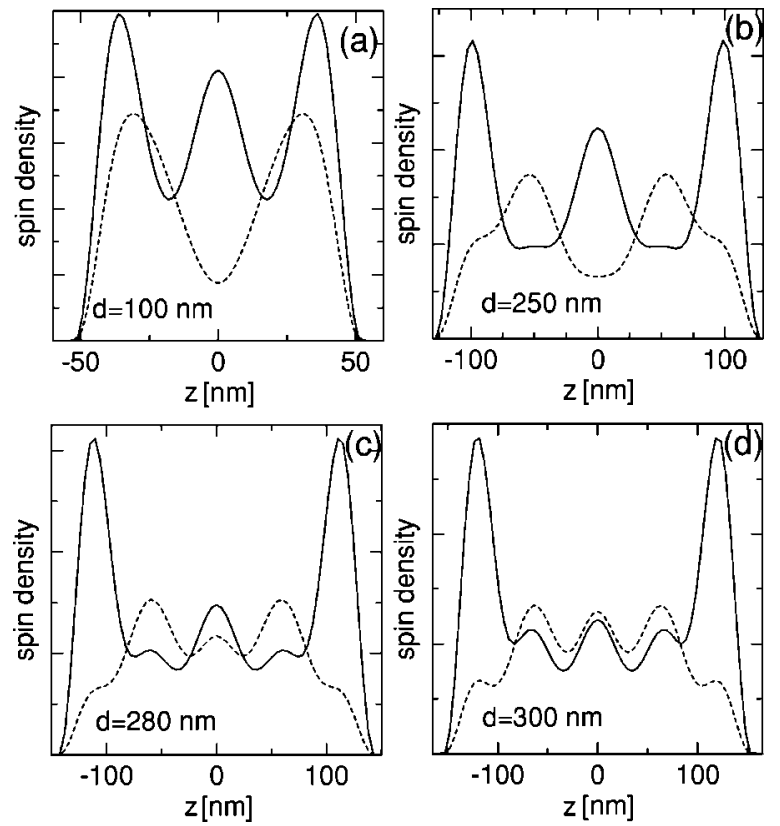

FIG. 9. Spin-up (solid lines) and spin-down (dashed lines) densities for the ground-state 5 -electron system $1 / 2+$ with $S_{z}=\hbar / 2$ for different system sizes.

real-space spin ordering with the spin orientation changing between the adjacent charge maxima vanishes.

Let us look at the spin distribution in the inner coordinates of the 5-electron $1 / 2+$ ground state. Figure 10(a) shows the PCF plots for $d=100 \mathrm{~nm}$. Electrons of the same spin as the fixed electron do not appear in its close neighborhood, but are more probable to be found at the center of the dot than electrons of opposite spin. Probability of finding an electron at the opposite side of the dot is independent of its spin. The spin order in this relatively small $\operatorname{dot}(d=100 \mathrm{~nm})$ is clearly short range which is similar as for the case of four electrons in a small dot [cf. Fig. 8(a)]. The PCF plots for opposite spins at the left end of the dot start to differentiate for $d$ $=200 \mathrm{~nm}$ [cf. Fig. 8(b)]. For $d=300 \mathrm{~nm}$, for which Wigner localization is observed [cf. Fig. 4(d)], the PCF plots show a long-range antiferromagnetic spin ordering. Notice the growth of the PCF plot for the same spin direction in the closest neighborhood of the fixed-position electron from $d$ $=200$ to $300 \mathrm{~nm}$ in Figs. 8(b) and 8(c). Pauli exclusion plays a less significant role for larger distances between the charge maxima.

Density-functional studies ${ }^{16,28}$ predict the appearance of interlocked waves of opposite spins in the laboratory frame for long quasi-one-dimensional dots. The appearance of the spin-density wave for even electron number amounts in spin symmetry breaking. Recently, ${ }^{16}$ it was found that for even $N$ the formation of the spin density wave in the density functional theory accompanies the Wigner crystallization. But in the present study we find that for the exact solution spin symmetry is conserved and Wigner crystallization is associated with the inner space spin ordering. In the exact solution the interlocked spin densities in the laboratory frame can only be observed for odd numbers of electrons, but the presented five-electron case shows that this effect is not necessarily related with Wigner crystallization. In the exact solution the electrons with opposite spins avoid one another in the inner space. A mean field approach can only account for this effect by symmetry breaking. The reason for the occurrence of spin symmetry breaking in the mean field approach for large single-dimensional dots are similar to the origin of the broken spatial symmetry mean field solutions for the magnetic field induced Wigner crystallization in circular structures. ${ }^{12}$

In large systems the spin-independent PCF plots become identical for all states degenerate with the ground state [cf. spin-independent PCFs for the four-electron degenerate $0+$ and 1- states in Figs. 8(b) and 8(c)]. This means that in Wigner-molecule states electrons avoid one another with the same efficiency independently of their spins. As a matter of fact this is the origin of the appearance of the ground state degeneracy in the Wigner molecule regime. One-dimensional Wigner molecules present pronounced magnetic properties related to the long-range spin ordering in the inner coordinate space. This ordering for different degenerate spin eigenstates may be typical for ferromagnetic, antiferromagnetic or even an other form of order. Due to the vanishing energy spacing between the different spin states the spin magnetic properties of Wigner molecules are of a very soft character. The Wigner molecules should be extremely susceptible to any spin-dependent interactions. In particular, even a weak additional effect promoting the spin-polarized phase can result in spin polarization of the system. A possible spin polarization of the one-dimensional electron gas has been found ${ }^{2}$ in transport measurements.

\section{CONCLUSIONS AND SUMMARY}

We have studied the ground and excited states of electron systems confined in quasi-one-dimensional quantum dots using an exact diagonalization approach. For large systems we found Wigner localization which appears not only in the
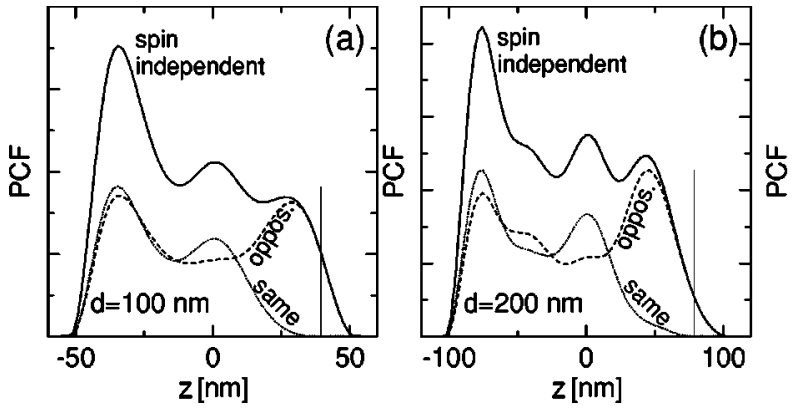

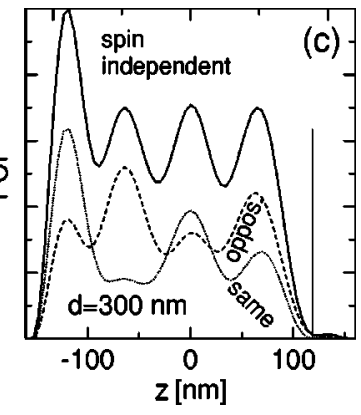

$035401-8$
FIG. 10. PCFs for five electrons in the state $1 / 2+$ for $d=100$ (a), 200 (b), and $300 \mathrm{~nm}$ (c). The position of the fixed electron is marked by a thin vertical line. Solid lines show the spinindependent PCF, dashed (dotted) lines show the opposite (same) spin PCF. 
ground state but also for several excited states which eventually leads to the degeneracy of the ground state in the large $d$ limit. We have considered spin and spatial parity of states forming Wigner molecules. We have shown that the parity of the spin-polarized state which forms a Wigner molecule is strictly determined by the number of electrons.

We have discussed the effect of a central attractive defect which destroys Wigner crystallization for an even number of electrons allowing more states to become degenerate with the ground state in the weak confinement limit. For odd electron numbers the central defect enhances the localization of the electron occupying the central position in the Wigner molecule and does not affect the ground state degeneracy.

We have investigated the spin-ordering effects associated with Wigner crystallization. We have found that for small dots the spatial spin ordering in the inner coordinates has a short-range character and results mainly from the Pauli exclusion principle. The long-range spatial spin order appears only in the Wigner molecule regime when the electrons occupy distinct sites within the quantum dot. We conclude that in one-dimensional quantum dots the Wigner crystallization is a necessary condition for the long range spin ordering to appear. We have identified the effect of spin symmetry breaking observed in the density functional theory as a tendency of the mean field method to mimic the internal-space spin ordering present in the exact solution for the Wigner molecule regime.

\section{ACKNOWLEDGMENTS}

This paper has been partly supported by the Polish Ministry of Scientific Research and Information Technology in the framework of solicited Grant No. PBZ-MIN-008/P03/ 2003, by the Flemish Science Foundation (FWO-V1), the Belgian Science Policy and the University of Antwerpen (VIS and GOA). B.S. was supported by the Foundation for Polish Science (FNP) and T.C. was partially supported by the Marie Curie Training Site Programme of the European Union.
${ }^{1}$ S. Tarucha, T. Honda, and T Saku, Solid State Commun. 94, 413 (1995).

${ }^{2}$ K. J. Thomas, J. T. Nicholls, M. Y. Simmons, M. Pepper, D. R. Mace, and D. A. Ritchie, Phys. Rev. Lett. 77, 135 (1996).

${ }^{3}$ A. Yacoby, H. L. Stormer, N. S. Wingreen, L. N. Pfeiffer, K. W. Baldwin, and K. W. West, Phys. Rev. Lett. 77, 4612 (1996).

${ }^{4}$ A. Bachtold, C. Strunk, J.-P. Salvetat, J.-M. Bonard, L. Forro, T. Nussbaumer, and C. Schonenberger, Nature (London) 397, 673 (1999).

${ }^{5}$ L. Jacak, P. Hawrylak, and A. Wójs, Quantum Dots (Springer, Berlin, 1998).

${ }^{6}$ S. Viefers, P. Koskinen, P. Singha Deo, and M. Manninen, Physica E (Amsterdam) 21, 1 (2004).

${ }^{7}$ M. S. Gudiksen, L. J. Lauhon, J. Wang, D. C. Smith, and C. M. Lieber, Nature (London) 415, 617 (2002).

${ }^{8}$ Y. Wu, R. Fan, and P. Yang, Nano Lett. 2, 83 (2002).

${ }^{9}$ M. T. Björk, B. J. Ohlsson, T. Sass, A. I. Persson, C. Thelander, M. H. Magnusson, K. Deppert, L. R. Wallenberg, and L. Samuelson, Nano Lett. 2, 82 (2002).

${ }^{10}$ F. P. Wigner, Phys. Rev. 46, 1002 (1934).

${ }^{11}$ R. Egger, W. Häusler, C. H. Mak, and H. Grabert, Phys. Rev. Lett. 82, 3320 (1999).

${ }^{12}$ S. M. Reimann and M. Manninen, Rev. Mod. Phys. 74, 1283 (2002).

${ }^{13}$ K. Jauregui, W. Häusler, and B. Kramer, Europhys. Lett. 24, 581 (1993).

${ }^{14}$ W. Häusler and B. Kramer, Phys. Rev. B 47, 16353 (1993).

${ }^{15}$ S. Bednarek, T. Chwiej, J. Adamowski, and B. Szafran, Phys. Rev. B 67, 205316 (2003).

${ }^{16}$ E. Räsanen, H. Saarikoski, V. N. Stavrou, A. Harju, M. J. Puska, and R. M. Nieminen, Phys. Rev. B 67, 235307 (2003).

${ }^{17}$ L. I. Glazman, I. M. Ruzin, and B. I. Shklovskii, Phys. Rev. B 45, 8454 (1992).
${ }^{18}$ H. J. Schulz, Phys. Rev. Lett. 71, 1864 (1993).

${ }^{19}$ F. Anfuso and S. Eggert, Phys. Rev. B 68, 241301 (2003).

${ }^{20}$ G. Piacente, I. V. Schweigert, J. J. Betouras, and F. M. Peeters, Solid State Commun. 128, 57 (2003).

${ }^{21}$ S. Bednarek, B. Szafran, T. Chwiej and J. Adamowski, Phys. Rev. B 68, 045328 (2003).

${ }^{22}$ M. Koskinen, M. Manninen, B. Mottelson, and S. M. Reimann, Phys. Rev. B 63, 205323 (2001).

${ }^{23}$ P. A. Maksym, Phys. Rev. B 53, 10871 (1996).

${ }^{24}$ C. G. Bao, W. Y. Ruan, and Y. Y. Liu, Phys. Rev. B 53, 10820 (1996).

${ }^{25}$ E. B. Kolomeisky and J. P. Straley, Rev. Mod. Phys. 68, 175 (1996).

${ }^{26}$ H. Tasaki, J. Phys.: Condens. Matter 10, 4353 (1998).

${ }^{27}$ E. Lieb and D. Mattis, Phys. Rev. 125, 164 (1962).

${ }^{28}$ S. M. Reimann, M. Koskinen, P. E. Lindelof, and M. Manninen, Physica E (Amsterdam) 2, 648 (1998).

${ }^{29}$ The approximate ground-state degeneracy of a $N$ electron system in large one-dimensional dot equals $2^{N}$ (taking into account the degeneracies with respect to the $z$ component of the total spin) as reported already in Ref. 14. For four and five electrons also higher-energy states for a given spin and parity quantum numbers (not discussed in the present paper) become degenerate with the ground state. We have determined the parity of these states. Namely, the four-electron ground state becomes degenerate with second $1-$ and $0+$ states and the five-electron ground state becomes degenerate with the second $1 / 2-, 3 / 2-, 1 / 2+$, and $3 / 2+$ symmetry states as well as with the third $1 / 2+$ state.

${ }^{30} \mathrm{~A}$ single determinant is sufficient to describe the spin-polarized Wigner molecule formed by $N$ electrons localizated around $N$ space sites. See also the discussion of the electron-electron correlation given in Ref. 15 . 